\title{
Single gyroid-structured metallic nanoporous spheres fabricated from double gyroid-forming block copolymers via templated electroless plating
}

\author{
Kai-Chieh Yang ${ }^{1}$, Cheng-Thai Yao ${ }^{1}$, Liang-Yu Huang ${ }^{2}$, Jing-Cherng Tsai ${ }^{2}$, Wei-Song Hung ${ }^{3,4}$, Han-Yu Hsueh ${ }^{5,6}$ and \\ Rong-Ming $\mathrm{Ho}^{1}$
}

\begin{abstract}
Herein, we suggest a methodology for the fabrication of well-defined metallic nanoporous spheres with single gyroid (SG) structure by simply using self-assembled diblock copolymer with double gyroid (DG) structure as a template for electroless plating. Note that owing to the consideration of thermodynamic stability, the self-assembly of diblock copolymers gives rise to a DG phase instead of an SG phase. By controlling the nucleus density for the reduction of Pd ions within the diblock copolymer template, SG-structured $\mathrm{Ni}$ can be easily fabricated through the nucleation and growth processes. Consequently, nanoporous Ni spheres with uniform pore sizes and high specific surface areas can be fabricated. Moreover, nanoporous Ni spheres with controlled microscale particle sizes can thus be obtained by controlling the reduction time for the growth of $\mathrm{Ni}$, which enables the feasibility of recyclability via magnetic fields. The combination of structural and morphological characteristics of the fabricated nanoporous Ni spheres make them appealing for use in a wide variety of applications, such as high-efficiency and well selectivity hydrogenation catalysts with recyclability due to their narrow pore size distributions, high specific surface areas, 3D curved surfaces, and controlled microscale particle sizes.
\end{abstract}

\section{Introduction}

Nanostructural and morphological designs of metallic materials have drawn much attention because of their effectiveness in tuning electronic, optical, and magnetic properties $^{1-10}$. In particular, the synthesis of nanoporous metals with controlled pore size and shape has become a main goal of extensive research on catalytic materials in recent years ${ }^{11-13}$. It is noted that highly active lowcoordinated atoms can be acquired from the curved surfaces of interconnected networks, which serve as excellent

\footnotetext{
Correspondence: Rong-Ming Ho (rmho@mx.nthu.edu.tw) or

Han-Yu Hsueh (hyhsueh@nchu.edu.tw)

'Department of Chemical Engineering, National Tsing Hua University, Hsinchu 30013 Taiwan, Republic of China

2Department of Chemical Engineering, National Chung Cheng University, Chiayi 62102 Taiwan, Republic of China
}

Full list of author information is available at the end of the article. catalytic sites for chemical reactions ${ }^{14-16}$. Nanoporous metals fabricated with manufacturing approaches such as dealloying usually appear as two-dimensional (2D) sheetlike structures ${ }^{1,17,18}$. It is essential to determine how to increase both the surface area to mass ratio and the density of catalytic sites of nanoporous metals to boost their surface activity performances over those of conventional 2D sheets. As a result, the fabrication of three-dimensional (3D) porous metals, particularly with nanoscale network textures, is in strong demand because of their large specific surface areas from the reduced pore sizes and self-supporting characteristics with low densities (i.e., lower required mass for equivalent catalytic efficiency). Therefore, the fabrication of 3D network catalysts has been dedicatedly conducted ${ }^{19}$. Note that highperformance catalysts are also recognized by their 
recyclability in addition to their catalytic efficiency. To recycle metallic catalysts, a filtering process is usually required to separate the metallic catalysts from the products. However, the filtering process is one of the most expensive procedures in industrial fabrication. One solution is to fabricate microscale metallic nanoporous spheres (MNSs) with magnetic properties for recycling purposes. Solutions with well-dispersed metallic spheres can be simply achieved by shearing due to the micrometer size of the spheres, which serves as the smallest size with saturation magnetization close to bulk ${ }^{20}$, gives the required dispersion for the intended reactions and provides feasibility for the collection of metallic catalysts after the reactions ${ }^{21}$. Although the reported properties and applications of MNSs have been promising, synthesizing monodispersed free-standing MNSs with superior catalytic performance and recyclability via a simple and cost-effective strategy remains a challenge ${ }^{22}$.

In recent decades, block copolymers (BCPs) have been extensively investigated because of their ability to selfassemble into one-dimensional (1D), 2D, and 3D periodic nanostructures with readily adjustable sizes, depending on their constituted compositions and molecular weights ${ }^{23}$. By taking advantage of the degradable characteristics of gyroid-forming BCPs, nanoporous polymers with wellordered network textures can be prepared by the preferential removal of constituted degradable components in $\mathrm{BCPs}^{24-31}$. Because well-ordered porous polymers contain continuous nanochannels, a variety of synthetic processes have been developed by using fabricated nanoporous polymers as templates for the fabrication of well-defined nanohybrids and inorganic metals with appealing properties after removal of the polymer template $^{32-35}$. Herein, we aim to suggest a facile approach for the fabrication of MNSs with network textures, which provide high porosities, controlled pore sizes, and narrow pore size distributions by exploiting nucleation and growth mechanisms for the reduction of metallic ions from templated electroless plates by using gyroidstructured templates from the self-assembly of diblock copolymers $^{36}$. The self-assembled gyroid phase from diblock copolymers is a pair of gyroid networks, wherein one is right-handed and the other is left-handed, that gives a thermodynamically stable morphology. Note that well-defined metallic nanonetworks have been fabricated via templated electroless plating in our previous work; however, our previous work fabricated shifting double gyroid (DG) network with a pair of right-handed and lefthanded networks, which resulted in a broadened pore size distribution after removal of the polymer template ${ }^{37,38}$. Moreover, the fix-bed type of fabricated nanonetworkstructured metallic materials hampered the catalytic performances of the intended chemical reactions in solutions, which resulted in the occurrence of catalytic poisoning and a lack of suspendability. With the use of a higher reduction potential material (i.e., Pd) as an initial nucleus for the reduction of $\mathrm{Ni}$ ions, it is feasible to acquire single gyroid (SG)-structured Ni from the DG-forming template. Following the growth of the reduced $\mathrm{Ni}$, a well-developed SG-structured Ni sphere with controlled pore size can be successfully fabricated; this solves the pore size broadening problem that occurs in templated synthesis using DG-structured templates after removal of the template, which is caused by network shifting. Additionally, the suspendability and recyclability characteristics of their micrometer-sized spherical textures are beneficial to homogeneous catalytic reactions in chemical solutions. With a narrow pore size distribution, high specific surface area, 3D curved surface, and controlled microscale particle size (i.e., the growth size of the reduced $\mathrm{Ni}$ ), the fabricated SG-structured Ni nanoporous sphere is an ideal candidate to serve as a high-efficiency recyclable catalyst, which is demonstrated in the catalytic hydrogenation of unsaturated organics and intended for unsaturated polymers.

\section{Materials and methods \\ Materials}

Polystyrene- $b$-poly(L-lactide) (PS-PLLA) was prepared by Atom transfer radical polymerization (ATRP) synthesis followed by ring-opening polymerization, whereas polystyrene- $b$-poly(dimethyl siloxane) (PS-PDMS) was prepared by anionic polymerization. As described in our previous works, PS-PLLA (molecular weight, $\mathrm{Mn}, \mathrm{PS}=$ $38,100 \mathrm{~g} \mathrm{~mol}^{-1}$, Mn, PLLA $=24,700 \mathrm{~g} \mathrm{~mol}^{-1}, Ð=1.23$, $f_{\text {PLLA }} \approx 0.35$, where $f_{\text {PLLA }}{ }^{\mathrm{v}}$ is the volume fraction of PLLA) and PS-PDMS (molecular weight, Mn, PS = $51,000 \mathrm{~g} \mathrm{~mol}^{-1}, \mathrm{Mn}, \mathrm{PDMS}=35,000 \mathrm{~g} \mathrm{~mol}^{-1}, Ð=1.03$, $f_{\text {PDMS }}{ }^{\mathrm{v}} \approx 0.42$, where $f_{\mathrm{PDMS}}{ }^{\mathrm{v}}$ is the volume fraction of PDMS) were used as materials for solution casting to fabricate PS templates with DG nanochannels. After hydrolytic treatment with a sodium hydroxide aqueous solution (2M), PLLA block can be selectively removed, which yields DG-structured PS with interconnected nanochannels and thru-pore characteristics (Fig S1). Similar porous PS can also be obtained by using selfassembled PS-PDMS (Fig S2(a)) for selective hydrofluoric acid (HF acid) etching of the PDMS block (Fig S2(b)).

\section{Fabrication of SG-structured MNSs via electroless plating}

To fabricate Ni nanoporous spheres, electroless plating was carried out through nucleation and growth mechanisms. For nucleation, the nanoporous PS templates were soaked in a low-concentration activating solution mixed with methanol $(45 \mathrm{~mL}), \mathrm{HCl}(1 \mathrm{~N}, 5 \mathrm{~mL})$, and $\mathrm{PdCl}_{2}$ $(0.004 \mathrm{~g})$ for several hours $(3-4 \mathrm{~h})$ followed by immersion in a reduction solution of hydrazinium hydroxide $(5 \mathrm{~mL})$ and methanol $(15 \mathrm{~mL})$. After nucleation, the template 
with the nucleus was soaked in a $\mathrm{Ni}$ bath $(0.2 \mathrm{~g}$ of nickel chloride $\left(\mathrm{NiCl}_{2} \cdot 6 \mathrm{H}_{2} \mathrm{O}\right)$ dissolved in a solution containing distilled water $(20 \mathrm{~mL})$, methanol $(20 \mathrm{~mL})$, hydrazinium hydroxide $(85 \%, 2 \mathrm{~mL})$, and ammonia $(3.5 \mathrm{~mL})$ ) to grow the Ni. To obtain the free-standing SG-structured Ni nanoporous sphere, the PS/Ni gyroid-structured nanohybrids were washed with either dichloromethane or tetrahydrofuran (THF) to remove the nanoporous PS template.

\section{Transmission electron microscopy}

To acquire transmission electron microscopy (TEM) images, an SG-structured Ni nanoporous sphere in a PS template was prepared by microtomy. A JEOL JEM-2100 LaB6 transmission electron microscope was used for TEM imaging at an accelerating voltage of $200 \mathrm{kV}$.

\section{Field-emission scanning electron microscopy}

To acquire field-emission scanning electron microscopy (FESEM) images, an SG-structured Ni powder was prepared after the PS template was removed. A JEOL JSM-6700 field-emission scanning electron microscope was used for FESEM imaging at accelerating voltages of $1.5 \mathrm{e} 3 \mathrm{keV}$.

\section{In situ small-angle $\mathrm{X}$-ray scattering}

In situ temperature-resolved small-angle X-ray scattering (SAXS) experiments were conducted at the synchrotron X-ray beam-line X27C at the National Synchrotron Radiation Research Center in Hsinchu, Taiwan, to examine the forming Ni texture in the PS/Ni nanohybrids.

\section{Wide-angle X-ray diffraction}

Wide-angle X-ray diffraction (WAXD) was conducted to examine the crystallinity of the SG-structured Ni. As shown in Fig S3, all of the diffractions can be indexed as face-centered cubic $\mathrm{Ni}$ with the lattice constant $a=3.52$ $\AA$, JCPDS card no. 4-856, corresponding to the reflections from the (111), (200), (220), (311), and (222) planes. Moreover, the characteristic peaks of $\mathrm{NiO}$ and $\mathrm{Ni}(\mathrm{OH})_{2}$ were not detected, which indicates that a well-defined crystalline structure of the $\mathrm{Ni}$ structure can be obtained under ambient conditions from templated electroless plating without oxidation.

\section{Demonstration of the recyclability of Ni nanoporous spheres}

As demonstrated in Video $\mathrm{S} 1$, the $\mathrm{Ni}$ nanoporous spheres in powder form used for the catalytic reaction of hydrogenation were dispersed in toluene to create a solution with $50 \mathrm{mg} \mathrm{Ni}$ catalyst in $15 \mathrm{~mL}$ toluene as a representative condition for the reaction. The $\mathrm{Ni}$ catalyst powders can be effectively collected by a regular magnet, and then the dispersion of the collected Ni powders can be simply achieved by ultrasonic agitation. The collection and dispersion of the Ni powders can be easily repeated without any limitation.

\section{Results and discussion \\ Synthesis and identification of DG-structured and SG- structured $\mathrm{Ni}$}

Figure 1 illustrates the experimental procedures for the fabrication of the MNSs in this study. As previously demonstrated by our laboratory, nanoporous PS with DGstructured nanochannels can be fabricated by either hydrolyzing self-assembled PS-PLLA or HF etching of PSPDMS, which can then be used as templates for the fabrication of DG-structured Ni through templated electroless plating ${ }^{32,39,40}$. With a completely pore-filled $\mathrm{Pd}^{2+}$ solution within the nanochannels of the PS template, homogeneously distributed Pd nuclei can be formed using hydrazine as the reduction agent. As shown in Fig. 1a, the formation of Pd nanoparticles from the reduction of $\mathrm{Pd}$ ions is carried out first, and then the Pd nanoparticles can serve as nuclei for the reduction of Ni ions to give DGstructured $\mathrm{Ni}$. In contrast, with a decrease in the nucleation density (i.e., the number of Pd nuclei in the template), as shown in Fig. 1b, SG-structured Ni can be fabricated due to the annihilation and growth of nickel simultaneous from neighboring nanochannels. Consequently, SG-structured $\mathrm{Ni}$ nanoporous spheres with controlled sphere sizes can be obtained by tuning the growth time of reduced $\mathrm{Ni}$ ions through the nucleation and growth processes.

Figure 2a, b show the TEM micrographs of the forming Pd nanoparticles in the PS template. As illustrated in Fig. 2a, Pd nanoparticles can be homogenously distributed in the PS template when the concentration of Pd ions in methanol is high (see below for details). In contrast, a significant reduction in the number of Pd nanoparticles can be found once the concentration of Pd ions in methanol is decreased to a certain level (see below for details); as exemplified in Fig. 2b, a single Pd nanoparticle can be observed from the viewing area. Subsequently, the reduction of the $\mathrm{Ni}$ ions using the $\mathrm{Pd}$ nanoparticles as catalytic sites can be carried out, which generates the formation of Ni network(s) through continuous growth within the gyroid-structured nanochannels. With a high density of Pd nanoparticles in the PS template, it is reasonable to expect the formation of two interdigitated networks. As shown in Fig. 2c, the [111] projection of the DG-structured PS/Ni nanohybrids can be clearly identified without staining, wherein the high mass-thickness contrast of Ni appears as the dark microdomains, whereas the PS is the bright matrix. In contrast, with a low density of Pd nanoparticles in the PS template, only one network will be formed, as evidenced from the [111] projection in which the hexagonally packing cell texture can be clearly 


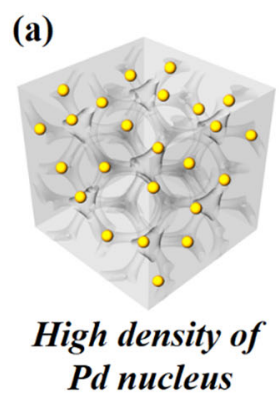

(b)

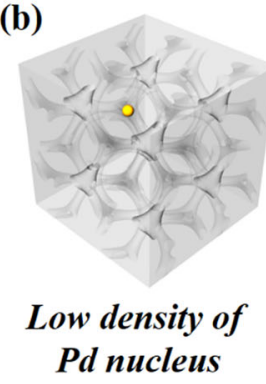

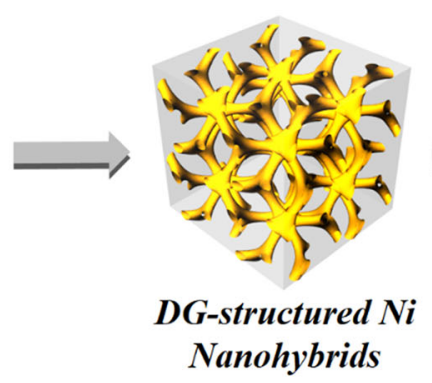
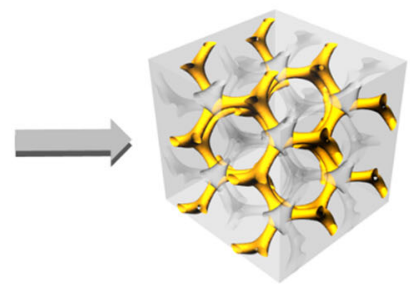

SG-structured Ni

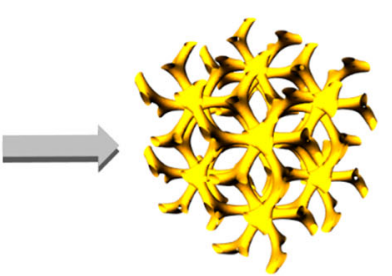

DG-structured Ni Nanomaterials

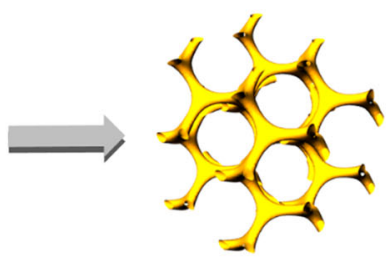

\section{SG-structured Ni Nanomaterials}

Fig. 1 Schematic illustration of the fabrication of SG- and DG-structured Ni from templated synthesis. Schematic illustrations of the fabrications of $\mathbf{a}$ double gyroid (DG)-structured and $\mathbf{b}$ single gyroid-structured Ni from templated electroless plating using a DG-structured polystyrene (PS) template from the self-assembly of either PS-b-poly(L-lactide) (PS-PLLA) followed by hydrolysis of PLLA block or PS-b-poly(dimethyl siloxane) (PS-PDMS) followed by HF etching of PDMS by controlling the nucleation and growth mechanisms for the reduction of $\mathrm{Ni}$ ions using Pd as a nucleus

observed (Fig. 2d). After removal of the PS template via dissolution with dichloromethane, the double-network texture of the DG-structured Ni can be clearly observed, as shown in Fig. 2e. In contrast, the single-network texture of the SG-structured Ni can be identified (Fig. 2f). Figure $2 \mathrm{~g}$ shows the 1D SAXS profile of the fabricated DG-structured PS/Ni. On the basis of the characteristic scattering reflections at the relative $q$ values of $\sqrt{ } 6$ and $\sqrt{ } 8$, the DG-structured PS/Ni nanohybrids (space group of Ia3d) can be identified macroscopically; the weak reflections are attributed to the strong scattering background from the forming $\mathrm{Ni}$ that smears the reflections from diffraction. Note that $q=4 \pi \lambda^{-1} \sin \theta$, where $2 \theta$ is the scattering angle. The corresponding 1D SAXS profile (Fig. 2h) further confirms the observed SG-structured Ni in which the characteristic reflections for a space group of $I 4_{1} 32$ are found at the relative $q$ values of $\sqrt{2}, \sqrt{ } 6, \sqrt{ } 8, \sqrt{ } 12$, $\sqrt{ } 18$, and $\sqrt{ } 30$. The appearances of the high-order reflections are attributed to the lower effects from scattering of the reduced $\mathrm{Ni}$ on the smearing of reflections. To further examine the structure of the fabricated $\mathrm{Ni}$, electron tomography (3D TEM) was carried out to directly visualize the forming texture in real space. As shown in Fig S4 (a), a 3D image of the SG-structured Ni in the PS matrix can be reconstructed from a set of $2 \mathrm{D}$ images at different tilt angles for projection (see SI for details). Fig S4(b) shows the corresponding simulated projection of the SG network.
As evidenced by the TEM observations and SAXS results, PS/Ni nanohybrids composed of SG-structured Ni in the PS template can be successfully fabricated using the template with low-density Pd nanoparticles as the nucleus to grow $\mathrm{Ni}$ via electroless plating. To further examine the forming $\mathrm{Ni}$ texture in the PS/Ni nanohybrids, in situ temperature-resolved SAXS experiments were conducted; the SG-structured PS/Ni nanohybrids were heated at a rate of $1{ }^{\circ} \mathrm{C} / \mathrm{min}$ from 50 to $185^{\circ} \mathrm{C}$ to trace the morphological evolution with a particular focus on the corresponding variations (Fig S5). There are no significant variations when the temperature is below $100^{\circ} \mathrm{C}$ (i.e., the $T_{\mathrm{g}}$ of PS), and the scattering result is contributed by the PS template with a DG-structured nanochannels (at relative $q$ values of $\sqrt{ } 6$ and $\sqrt{ } 8$ ) and the SG-structured $\mathrm{Ni}$ with a single-network texture (at relative $q$ values of $\sqrt{ } 2$, $\sqrt{6}$, and $\sqrt{ } 8$ ). Interestingly, once the temperature rises above $100^{\circ} \mathrm{C}$, there are obvious changes in the reflections of $\sqrt{6}$ and $\sqrt{ } 8$, which experience substantial intensity reductions. When the temperature increases above $105^{\circ}$ $\mathrm{C}$, where the PS template will be greatly softened and cause the collapse of the template texture (as evidenced by SEM observations; results not shown), the characteristic scattering reflection at the low $q$ region (i.e., the $\sqrt{2}$ reflection) can be clearly identified from the reflections of the $\sqrt{6}$ and $\sqrt{ } 8$ and becomes the primary peak in terms of scattering intensity. Note that compared to the reflections of $\sqrt{6}$ and $\sqrt{ } 8$, the intensity of the $\sqrt{2}$ peak almost remains 

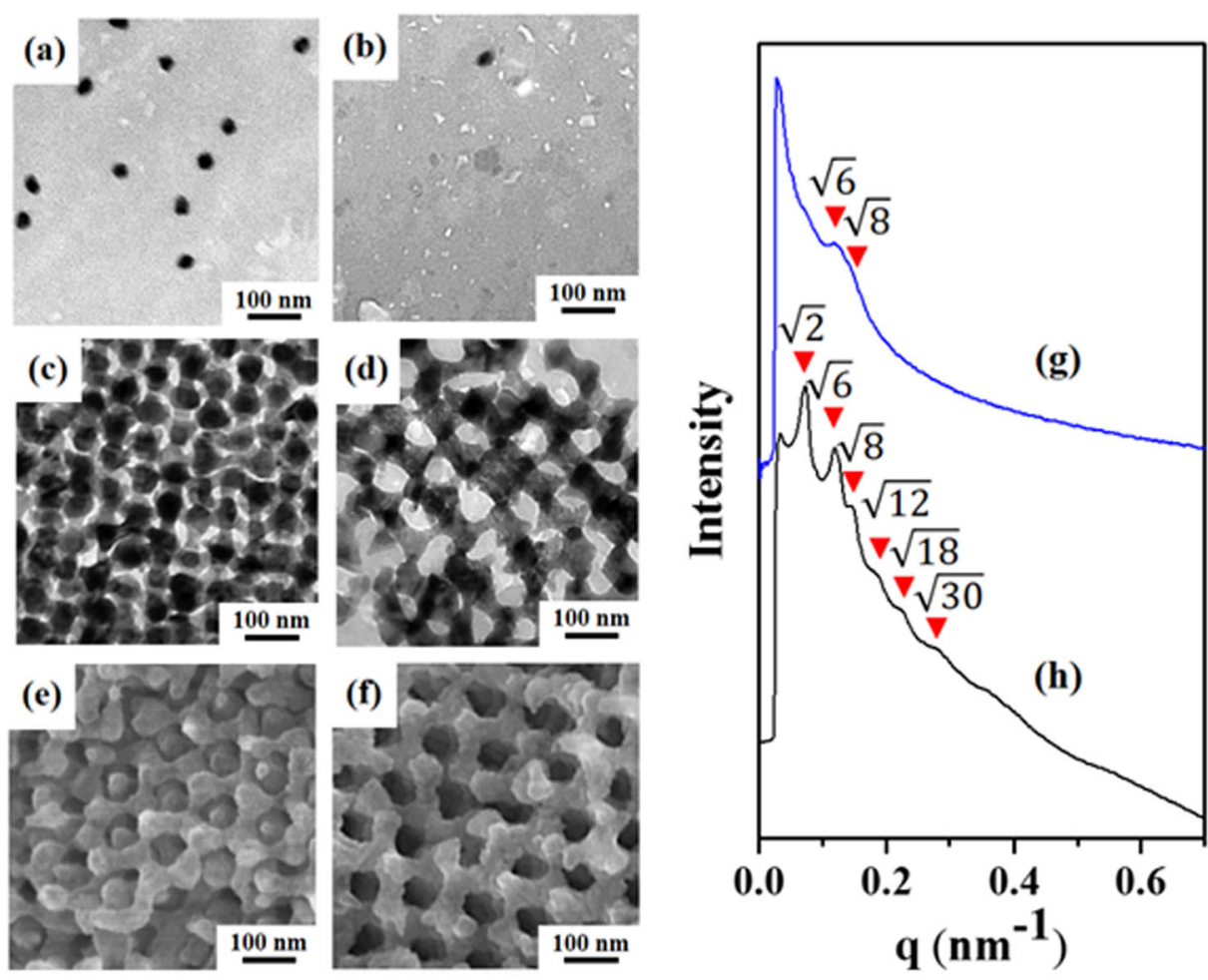

Fig. 2 TEM, SEM and SAXS results of fabricated SG- and DG-structured Ni. Transmission electron microscopy (TEM) micrographs of the Pd nucleus in the polystyrene (PS) template from templated electroless plating using a a high concentration (840 ppm) and $\mathbf{b}$ low concentration (120 ppm) of Pd ions in methanol for reduction. The [111] projected TEM micrographs of Ni with $\mathbf{c}$ double gyroid (DG) and $\mathbf{d}$ single gyroid (SG) texture in PS/Ni nanohybrids after templated electroless plating. Field-emission scanning electron microscopy micrographs of $\mathbf{e}$ DG-structured and $\mathbf{f} \mathrm{SG}$-structured Ni after removal of the PS template by washing with dichloromethane. $\mathbf{g}$, $\mathbf{h}$ One-dimensional small-angle X-ray scattering profiles of $\mathbf{c}$ and $\mathbf{d}$, respectively

constant regardless of the thermal treatment. These results suggest that the thermal treatment gives rise to the softening of the PS template with DG texture, which results in the weakening of the scattering from the DGstructured PS template due to its draining from the selfsupporting Ni network, while the appearance of isolated SG-structured Ni gives a characteristic scattering profile at the relative $q$ values of $\sqrt{2}, \sqrt{6}$, and $\sqrt{ } 8$. As a result, a successful approach for the fabrication of SG-structured $\mathrm{Ni}$ can be achieved by simply controlling the nucleation density of Pd nanoparticles for the reduction of the $\mathrm{Ni}$ via templated electroless plating.

To further examine the nucleation and growth mechanism of the templated electroless plating, methanol solutions with different concentrations of Pd ions, 12, 120, 360,600 , and $840 \mathrm{ppm}$, were prepared for the reduction of the Pd ions in the PS template. Figure 3a shows the SAXS profiles of the $\mathrm{PS} / \mathrm{Ni}$ nanohybrids. In contrast to the nanoporous PS, the low- $q$ reflection at the relative $q$ value of $\sqrt{2}$ can be easily recognized from the $\sqrt{6}$ and $\sqrt{8}$ reflections even though the concentration of Pd ions is as low as $12 \mathrm{ppm}$; this phenomenon illustrates that the scattering intensity from the forming SG-structured $\mathrm{Ni}$ in the PS template (Fig. 3b) is significant due to the large mass-thickness contrast of the $\mathrm{Ni}$ from the PS. With further increasing the Pd ion concentration (e.g., 120 ppm), the intensity of the $\sqrt{ } 2$ reflection can be gradually enhanced compared to those of $\sqrt{6}$ and $\sqrt{ } 8$; the observed variations are in agreement with suggested the nucleation and growth mechanism. Nevertheless, once the concentration of the Pd ions reaches a critical amount (e.g., $360 \mathrm{ppm}$ ), the intensity of the $\sqrt{2}$ reflection will be equivalent to that of $\sqrt{ } 6$, which indicates that the reduced Pd ions in the PS template may form the Pd nanoparticles in the neighboring nanochannels that results in the formation of DG in some regions of the template; this phenomenon is evidenced by the TEM observations (Fig. 3c). The TEM micrographs show biphasic morphologies with SG- and DG-structured Ni in the PS template at which the DG-structured $\mathrm{Ni}$ is marked with the red dashed circle. Subsequently, as the concentration of $\mathrm{Pd}$ ions increases to a certain amount (e.g., $600 \mathrm{ppm}$ ), a recognizable reduction in the intensities of the reflections can be found due to the significant scattering from the forming Ni that smears out the diffraction results of the ordered structure of the PS/Ni nanohybrids. With the 

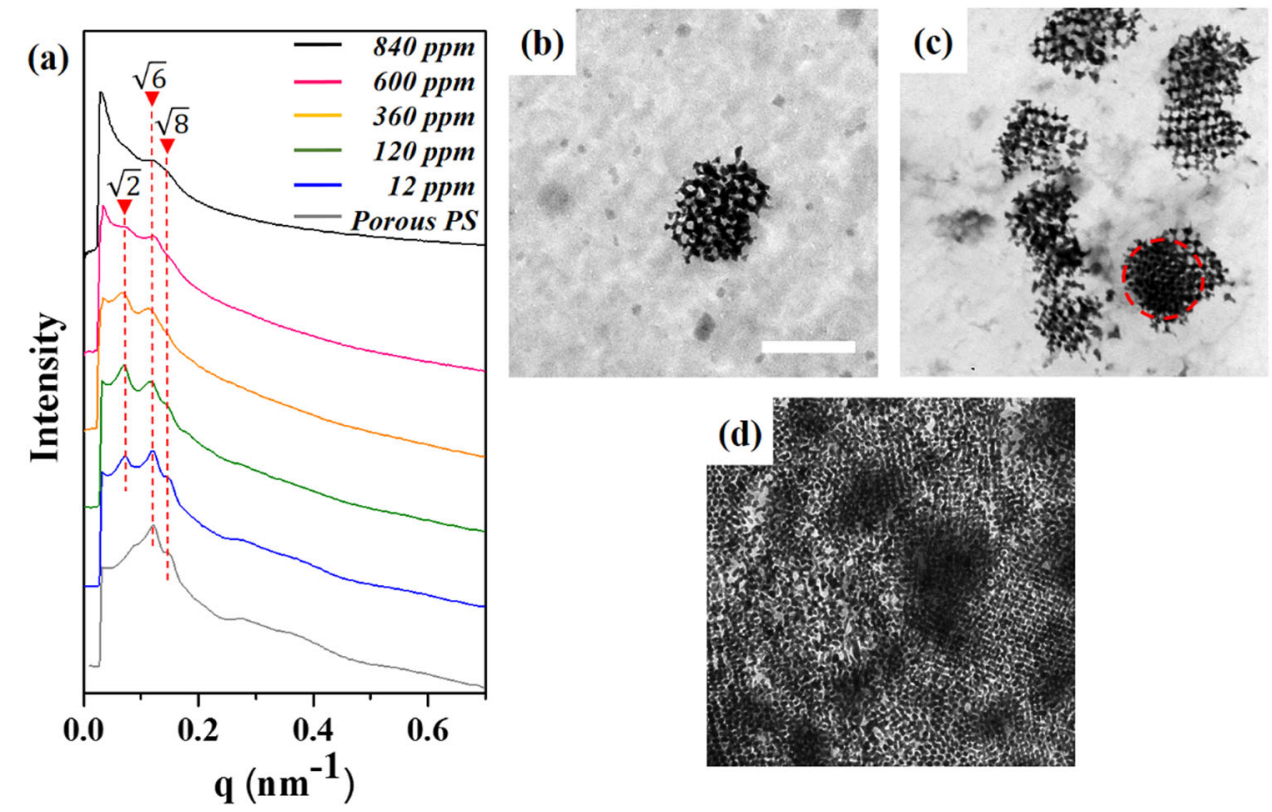

Fig. 3 Pd ion concentration effects on SG-structured Ni fabricated. a One-dimensional small-angle X-ray scattering profiles of the polystyrene (PS)/Ni nanohybrids with gyroid-structured Ni from templated electroless plating using nanoporous PS as a template and reduced Pd as the nucleus at different concentrations of Pd ions (milligrams of Pd ions per liter of solution). Transmission electron microscopy micrographs of PS/Ni nanohybrids with reduced Pd nuclei at b 12, c 360, and d 840 ppm Pd ion concentrations, which yield single gyroid (SG)-structured Ni, SG-structured Ni mixed with double gyroid (DG)-structured Ni (as marked in dashed circle), and DG-structured Ni in the PS matrix, respectively. All images have the same magnification, and the scale bar is $500 \mathrm{~nm}$

introduction of a large number of Pd ions (e.g., $860 \mathrm{ppm}$ ) for reduction, it is reasonable to expect the formation of DG-structured $\mathrm{Ni}$ in the PS template in which the Pd clusters are homogeneously distributed in the PS matrix, which generates the DG-structured $\mathrm{Ni}$ in the nanohybrids, as evidenced by Fig. 3d. Namely, the formation of a well-distributed Pd nucleus in two independent networks of a DG-structured PS template gives rise to the reduced $\mathrm{Ni}$ ions to fill the nanochannels of the PS template completely through templated electroless plating. Consequently, due to the large scattering from the forming $\mathrm{Ni}$, the scattering profiles of the nanohybrids in Fig. 3a become less identifiable. On the basis of the morphological observations examined above, it is feasible to tune the formation of SG-structured Ni from the uniformly dispersed Pd nucleus in the DG-structured PS template by controlling the concentration of $\mathrm{Pd}$ ions introduced, which further demonstrates that the formation of the reduced $\mathrm{Ni}$ is indeed conducted by the nucleation and growth mechanisms.

\section{Controlled growth of spherical particle size with adjustable pore size}

Figure 4 shows a low-magnification FESEM micrograph of an SG-structured Ni nanoporous sphere in which the SG-structured Ni network with a nanoscale uniform frame size and a microscale spherical texture can be clearly recognized after removal of the template. Moreover, a large number of micrometer-sized SG-structured $\mathrm{Ni}$ nanoporous spheres can be successfully obtained, which gives the potential for large-scale fabrication of $\mathrm{Ni}$ nanoporous spheres with micrometer-sized particles for various applications. The inset image of Fig. 4a shows a photograph of the SG-structured $\mathrm{Ni}$ in a brown powder form, which further demonstrates the fabrication of a great number of nanoporous $\mathrm{Ni}$ spheres in the powders. Figure $4 \mathrm{~b}$ shows a high-magnification image of the fabricated Ni nanoporous sphere in which a well-ordered SG texture can be clearly identified; similar morphological results can be observed in all of the nanoporous $\mathrm{Ni}$ spheres fabricated above. The crystalline structure of the SG-structured Ni was identified by a WAXD experiment (Fig S3). As a result, the sphere size of the SG-structured $\mathrm{Ni}$ can be well-controlled through the growth process by placing the PS template, which contains a lowconcentration Pd ions, in the solution for different growth times. To acquire systematic analysis on the growth of the $\mathrm{Ni}$ from templated electroless plating, morphological observations from SEM were conducted.

As shown in Fig. $5 \mathrm{a}-\mathrm{d}$, it is reasonable to observe the increase in the sphere size with respect to the reaction time (i.e., the growth time). The sphere size is approximately $320 \mathrm{~nm}$ after $3 \mathrm{~h}$ of reaction and gradually increases to approximately $760 \mathrm{~nm}$ after $7.5 \mathrm{~h}$. On the 


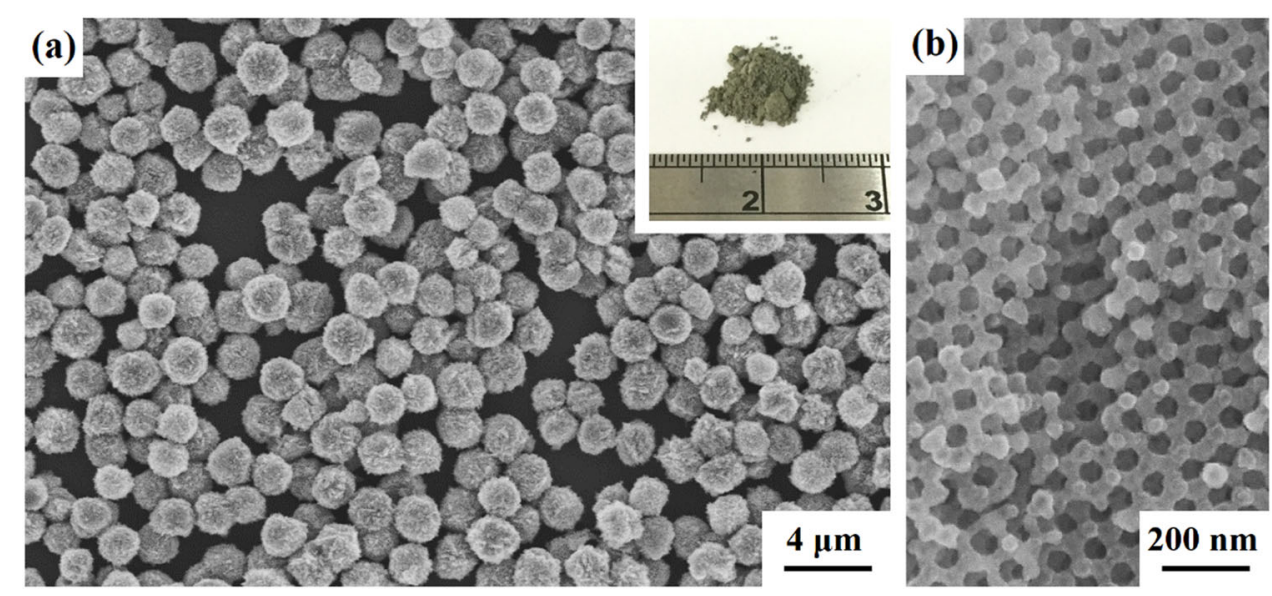

Fig. 4 Fabricated SG-structured Ni spheres with power form. a Low-magnification scanning electron microscopy micrograph and $\mathbf{b}$ [111] projection plane of single gyroid (SG)-structured Ni nanoporous spheres from templated electroless plating using nanoporous polystyrene as a template. The inset image shows a photograph of the SG-structured Ni nanoporous spheres in powder form
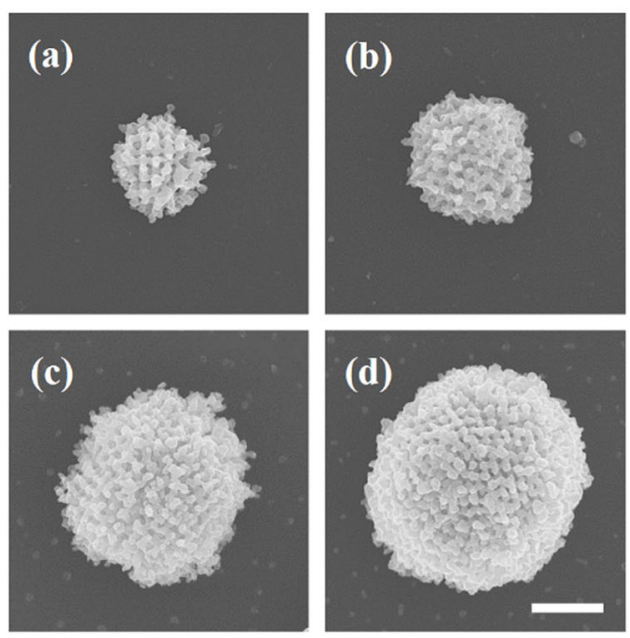

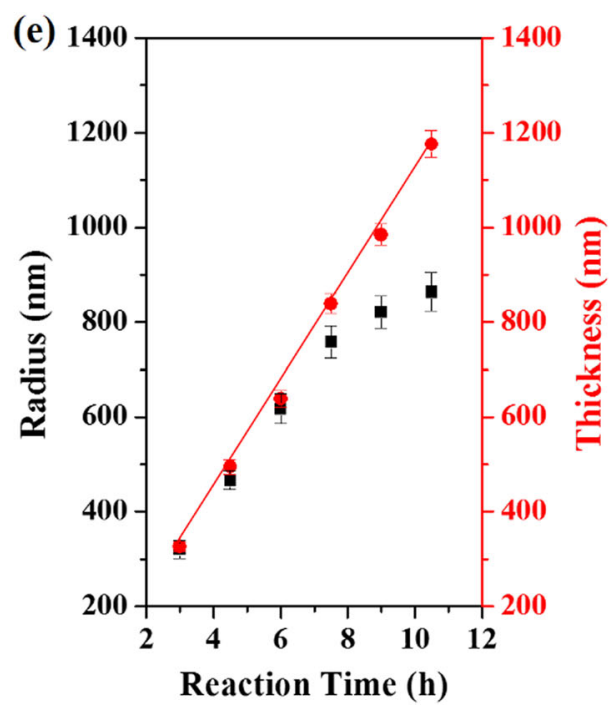

Fig. 5 Size control of SG-structured Ni sphere by growth time. Scanning electron microscopy micrographs of the single gyroid (SG)-structured Ni at different growth times for Ni reduction: $\mathbf{a} 3 \mathbf{h} ; \mathbf{b} 4.5 \mathrm{~h} ; \mathbf{c} 6 \mathrm{~h}$; and $\mathbf{d} 7.5 \mathrm{~h}$. All images have the same magnification, and the scale bar is $500 \mathrm{~nm}$. e Plot of the thicknesses of reduced $\mathrm{Ni}$ on Ni plates from electroless plating (red dot) and the particle radii of the SG-structured Ni (black dot) fabricated via templated electroless plating versus reduction time for Ni deposition

basis of the statistical calculation for the average particle size from the SEM micrographs, the growth rate can be estimated; as shown in Fig. 5e (black line), a linear relationship between the particle size and the reaction time can be found when the reaction time is $<7.5 \mathrm{~h}$, which suggests a typical growth pattern for the growth mechanism of heterogeneous nucleation initiating from the forming Pd nucleus. However, the growth rate of the SG-structured $\mathrm{Ni}$ nanoporous sphere experiences a reduction once the reaction time is $>7.5 \mathrm{~h}$. To further understand the cause of the reduction in growth rate, a control experiment was designed by using a uniform $\mathrm{Ni}$ plate for electroless plating to trace the growth rate of $\mathrm{Ni}$ deposition for longer reaction times. A silicon wafer with a surface roughness $<5 \mathrm{~nm}$ was coated with a smooth $\mathrm{Ni}$ thin film via sputtering and then immersed in the electroless plating bath for $\mathrm{Ni}$ deposition under the same conditions as the SG-structured Ni fabricated from templated electroless plating. The deposition rate of the $\mathrm{Ni}$ plate, $\mathrm{rp}(\mathrm{nm} / \mathrm{h})$, can be calculated from the weight gain of 

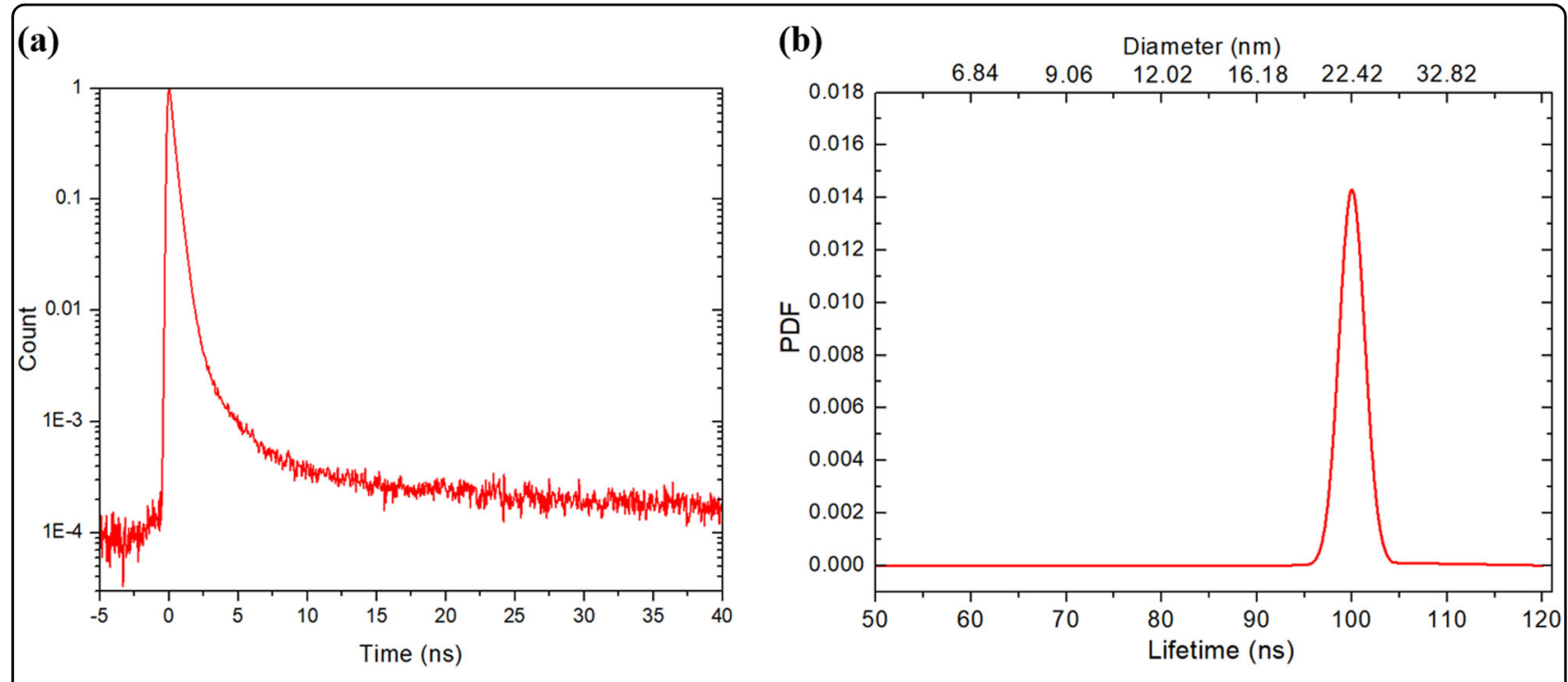

Fig. 6 Pore size determination of SG-structured Ni sphere using positron annihilation. a Age-momentum correlation spectrum ( $3 \gamma$ annihilation in the range of 340-490 keV) for the single gyroid (SG)-structured Ni powder. b Pore size distribution calculation by positron annihilation spectroscopy using the longest o-Ps lifetime obtained from age-momentum correlation

the Ni plate before and after Ni deposition via electroless plating using the following equation: $\mathrm{rp}=\omega \times 107 / \sigma A t$, where $\omega, \sigma, A$, and $t$ represent the weight increment (g), deposit density $\left(\mathrm{g} / \mathrm{cm}^{3}\right)$, substrate surface area $\left(\mathrm{cm}^{2}\right)$, and deposition time (h), respectively. The experimental values recorded in this study were the average values of more than five measurement results. As shown in Fig. 5e (red line), the film thickness constantly increases from approximately 330 to $1180 \mathrm{~nm}$ as the reaction time increases from 3 to $10.5 \mathrm{~h}$, which is a linear relationship that is nearly equivalent to that from templated electroless plating. However, in contrast to the templated electroless plating, there is no reduction in the growth rate for the control experiment. We speculate that the reduction in the growth rate during the templated electroless plating is attributed to the depletion of the $\mathrm{Ni}$ ions, while the blocking of $\mathrm{Ni}$ ions occurs due to the formation of $\mathrm{Ni}$. To further demonstrate the feasibility of the developed platform technology, PS-PLLA or PS-PDMS with different molecular weights and volume fractions were used to prepare $\mathrm{Ni}$ nanoporous spheres with SG-structured textures.

The control of the lattice constant for the SG and the corresponding filling ratios of the metallic nanoporous materials are critical to the expected properties for practical applications. To further demonstrate the feasibility of the developed platform technology for the preparation of Ni nanoporous spheres with SG-structured textures, the lattice constant of the SG and the corresponding filling ratio of the nanoporous Ni DG-structured PS-PLLA or PS-PMDS with different molecular weights and volume fractions were used to induce variations in the lattice constant of the SG-structured $\mathrm{Ni}$ and filling ratios of the fabricated nanoporous Ni. By using PS-PLLA as an example, on the basis of the SAXS results (Fig S6), the interdomain spacing of (110) $)_{\mathrm{SG}}$ for SG-structured Ni was determined to be approximately 113.0 and $87.1 \mathrm{~nm}$ from the primary reflection (blue and black lines). The lattice constants of SG-structured Ni can be calculated as 159.8 and $123.2 \mathrm{~nm}$. The filling ratios of SG-structured $\mathrm{Ni}$ with one single network can also be obtained as 21.0 and $17.5 \%$ from presynthesized PS templates with volume fractions of 42 and 35\%. Similar to the PS-PLLA demonstrated above, the lattice constant for the PS-PDMS can be sufficiently tuned through precision synthesis (Fig S6; see SI for details).

Positron annihilation spectroscopy was used to determine the pore size distribution of the fabricated SGstructured $\mathrm{Ni}$. A total of $50 \mathrm{mCi}$ of ${ }^{22} \mathrm{Na}$ was used as a positron source for the analysis. After implantation of the positron into the SG-structured Ni nanoporous spheres in powders under ambient conditions, the lifetime of the positron was detected (Fig. 6a). Within the lifetime being detected, the distribution of the longest o-Ps lifetime (Fig. 6a) was fitted to obtain the pick-off annihilation rate of o-Ps $\left(\mathrm{ns}^{-1}\right)\left(\lambda^{\mathrm{R}}\right)$ based on the age-momentum correlation technique combined with the selection of the $3 \gamma$ annihilation located in the range of 340-490 keV analyzed by using the PATFIT program. Consequently, the pore size of the fabricated SG-structured nickel was calculated based on the following equations in which the relationship between $\lambda^{\mathrm{R}}$ and volume radius $(R)$ was derived by 
Table 1 SG-structured Ni for the demonstration of high catalysis performance

\begin{tabular}{|c|c|c|c|c|c|c|c|}
\hline Entries & $\begin{array}{l}\text { Toluene/cyclohexene } \\
{[\mathrm{mL}]}\end{array}$ & $P$ [bar] & Temp. $\left[{ }^{\circ} \mathrm{C}\right]$ & Time $[\mathrm{h}]$ & $\begin{array}{l}\text { Cat. weight } \\
\text { [mg] }\end{array}$ & $\begin{array}{l}\text { Conversion for toluene/ } \\
\text { cyclohexene [\%] }\end{array}$ & $\begin{array}{l}\text { Toluene/cyclohexene turnover } \\
{\left[\mathrm{mol} \times \mathrm{cat} \mathrm{mol}^{-1} \times \mathrm{h}^{-1}\right]}\end{array}$ \\
\hline $1^{\mathrm{a}}$ & $3 / 0$ & 50 & 200 & 4 & 17 & 97\%/- & $72 /-$ \\
\hline $2^{\mathrm{b}}$ & $1.5 / 1.5$ & 50 & 80 & 4 & 17 & $3.8 \% / 78 \%$ & $2.7 / 20$ \\
\hline $3^{c}$ & $3 / 0$ & 50 & 200 & 4 & 17 & $70 \% /-$ & $50 /-$ \\
\hline
\end{tabular}

SG single gyroid

${ }^{a}$ SG-structured Ni hydrogenation for toluene

${ }^{\mathrm{b}} \mathrm{SG}$-structured $\mathrm{Ni}$ selective hydrogenation for unsaturated bond and aromatic ring

${ }^{c}$ Raney Ni hydrogenation for toluene

Tao and co-workers and further modified to Eq. (1) to include $3 \gamma$ from the o-PS annihilation to calculate pore sizes larger than $1 \mathrm{~nm}$ with $\Delta R=16.56 \mathrm{~nm}, R_{\mathrm{a}}=0.8 \mathrm{~nm}$ and $b=0.55^{41,42}$. Figure $6 \mathrm{~b}$ shows the calculated pore size distribution in which the full width at half of maximum $\left(D_{\mathrm{fwhm}}\right)$ can be as low as $3.7 \mathrm{~ns}$, which indicates that the SG-structured nickel possesses a uniform pore size of approximately $22.4 \mathrm{~nm}$; this result is in agreement with the observed pore size from Fig. 4, in which the pores should be well connected.

$$
\lambda^{R}=\left\{\begin{array}{c}
\lambda_{2 \gamma}^{R_{a}}\left[1-\left(\frac{R-R_{a}}{R+\Delta R}\right)^{b}\right]+\lambda_{3 \gamma} \ldots\left(R \geq R_{a}\right) \\
\lambda_{2 \gamma}^{R_{a}}+\lambda_{3 \gamma} \ldots \ldots \ldots \ldots \ldots \ldots \ldots\left(R<R_{a}\right)
\end{array}\right\}
$$

\section{Demonstration of using SG-structured MNSs for applications}

Due to its uniform nanoscale pore size, network texture, and controlled microscale sphere, the fabricated nanoporous $\mathrm{Ni}$ sphere is appealing for use in a wide variety of applications; herein, we aim to exploit it as a highefficiency hydrogenation catalyst as an exemplary application. Note that $\mathrm{Ni}$ metal is a commonly used heterogeneous catalyst in hydrogenation processes. In heterogeneous catalytic hydrogenation processes, efficient catalysts should provide high catalytic activity and excellent recyclability. For catalytic activity, the efficiency depends closely on the specific surface area and the kinetics of the mass transport process. Ni reduction by electroless plating is known to be organized in the facecentered cubic (fcc) crystalline phase, as evidenced by the reflections of (110), (220), and (200) in WAXD (Fig S3). A tight packing of atoms in the fcc lattice makes the reactants easily absorb on the surface of Ni. Most importantly, with the well-defined SG structure containing extremely high specific surface area, it is reasonable to expect high performance on the hydrogenation reaction. Furthermore, the continuous network structure of the SG with specific curvature and uniform pore size would definitely lower the limitation for mass transport of the reactants within the nanochannels, which provides the effectiveness of the surface area inside the $\mathrm{Ni}$ nanoporous sphere and prevents the unnecessary blocking caused by regions with smaller pore size; moreover, such a situation might occur in DG-structured Ni due to the shifting of networks after removal of the PS template ${ }^{43}$. The catalytic activity of the SG-structured Ni nanoporous sphere was demonstrated by hydrogenation of the aromatic ring (i.e., toluene) under regular reaction conditions (60 bar and $200{ }^{\circ} \mathrm{C}$ ), in which the conversion was approximately $97 \%$; in contrast, the conversion by Raney nickel under the same conditions was approximately $70 \%$. The respective turnover frequencies are listed in Table 1. Accordingly, the catalytic efficiency of the fabricated SG-structured $\mathrm{Ni}$ porous sphere performs were better than that of the commonly used Ni catalyst in industry (Raney nickel with a nickel composition of 61\%). Moreover, the SG-structured Ni with high efficiency possesses high selectivity for hydrogenation between cyclohexene and toluene. In contrast to noble metal catalysts, such as palladium and platinum, at low reaction temperatures (for instance, 50 bar and $80^{\circ} \mathrm{C}$ ), the SG-structured $\mathrm{Ni}$ could selectively hydrogenate the unsaturated double bond of cyclohexene without hydrogenating the aromatic ring of toluene (see Table 1 for details). Palladium and platinum are well-known highefficiency catalysts, and the hydrogenation of toluene and organics with unsaturated double-bonds will simultaneously occur in low-temperature reaction conditions $^{44,45}$. As demonstrated above, the SG-structured Ni nanoporous sphere with high activity and excellent selectivity indeed meets the criteria of high efficiency catalysis. Most importantly, for a cost-effective process, it is essential to possess catalytic recyclability; owing to the robust structure of the SG continuous network, the structure would be maintained after the hydrogenation reaction, which could be reused without a huge decay in efficiency after many cycles. Additionally, due to their microscale dimensions, the SG-structured Ni nanoporous spheres can be simply suspended in the solution for hydrogenation reaction and then simply recycled by using 
a magnet to repeatedly collect the spheres by applying a magnetic field and then dispersing the spheres in the suspension by stirring (see Video S1 for demonstration). Note that the SG-structured Ni nanoporous sphere is safe in the atmosphere, while Raney nickel, a fine grain of nickel-aluminum alloy that has a low density due to its incorporation of hydrogen, is flammable. Nickelaluminum alloy is a porous structure with high efficiency after the activation process during fabrication that simultaneously fills hydrogen into its pores, which results in low density and flammability. Therefore, Raney ${ }^{\circledR}$ nickel should be handled in an inert atmosphere to prevent the ignition problem that often causes severe problems in safety.

\section{Conclusions}

In conclusion, MNSs with SG structures can be successfully fabricated by using a DG-structured PS template for electroless plating through nucleation and growth mechanisms using a low nucleus density strategy for reduction. Furthermore, by controlling the deposition time for the reduction of $\mathrm{Ni}$, the sphere sizes of the MNSs can be fine-tuned. Consequently, free-standing SGstructured $\mathrm{Ni}$ spheres with high specific surface areas, uniform pore sizes, and microscale particle sizes can be obtained. As exemplified in this study, the fabricated $\mathrm{Ni}$ nanoporous sphere can serve as a high-activity catalyst for the hydrogenation process with good recyclability. As a result, the fabricated SG-structured $\mathrm{Ni}$ nanoporous spheres are appealing in a wide variety of applications because of their network shapes, robust properties, single networks, uniform nanoscale pore sizes, and microscale particle sizes, which give them excellent recyclability via magnetic fields.

\section{Acknowledgements}

The authors would like to thank the National Science Council of the Republic of China, Taiwan, for financially supporting this research under Contract No. Grant NSC 106-2119-M-007-010-, and the National Synchrotron Radiation Research Center (NSRRC) for its assistance in the synchrotron SAXS experiments. This work was supported in part by the Ministry of Education, Taiwan, R.O.C., under the Higher Education Sprout Project.

\section{Author details}

'Department of Chemical Engineering, National Tsing Hua University, Hsinchu 30013 Taiwan, Republic of China. ${ }^{2}$ Department of Chemical Engineering, National Chung Cheng University, Chiayi 62102 Taiwan, Republic of China. ${ }^{3}$ Department of Chemical Engineering and R\&D Center for Membrane Technology, Chung Yuan University, Taoyuan, Taiwan, Republic of China. ${ }^{4}$ Graduate Institute of Applied Science and Technology, National Taiwan University of Science and Technology, Taipei 10607 Taiwan, Republic of China. ${ }^{5}$ Department of Materials Science and Engineering, National Chung Hsing University, Taichung 40227 Taiwan, Republic of China. ${ }^{6}$ Innovation and Development Center of Sustainable Agriculture (IDCSA), National Chung Hsing University, Taichung 40227 Taiwan, Republic of China

\section{Publisher's note}

Springer Nature remains neutral with regard to jurisdictional claims in published maps and institutional affiliations.

Supplementary information is available for this paper at https://doi.org/ 10.1038/s41427-019-0108-z.

Received: 12 October 2018 Revised: 23 November 2018 Accepted: 29 November 2018.

Published online: 20 February 2019

\section{References}

1. Erlebacher, J., Aziz, M. J., Karma, A., Dimitrov, N. \& Sieradzki, K. Evolution of nanoporosity in dealloying. Nature 410, 450 (2001).

2. $Y u$, J. et al. Nanoporous metals by dealloying multicomponent metallic glasses. Chem. Mater. 20, 4548 (2008).

3. Kang, B. \& Ceder, G. Battery materials for ultrafast charging and discharging. Nature 458, 190 (2009).

4. Rolison, D. R. et al. Multifunctional $3 \mathrm{D}$ nanoarchitectures for energy storage and conversion. Chem. Soc. Rev. 38, 226 (2009).

5. Xia, Y., Xiong, Y., Lim, B. \& Skrabalak, S. E. Shape-controlled synthesis of metal nanocrystals: simple chemistry meets complex physics? Angew. Chem. Int. Ed. 48, 60 (2009)

6. Krishna, K. S., Sandeep, C. S. S., Philip, R. \& Eswaramoorthy, M. Mixing does the magic: a rapid synthesis of high surface area noble metal nanosponges showing broadband nonlinear optical response. ACS Nano 4, 2681 (2010)

7. Tappan, B. C., Steiner, S. A. \& Luther, E. P. Nanoporous metal foams. Angew. Chem. Int. Ed. 49, 4544 (2010).

8. Huang, $X$. et al. Palladium-based nanostructures with highly porous features and perpendicular pore channels as enhanced organic catalysts. Angew. Chem. Int. Ed. 52, 2520 (2013).

9. Qi, Z. \& Weissmüller, J. Hierarchical nested-network nanostructure by dealloying. ACS Nano 7, 5948 (2013).

10. Dixon, M. C. et al. Preparation, structure, and optical properties of nanoporous gold thin films. Langmuir 23, 2414 (2007).

11. Zhang, J. \& Li, C. M. Nanoporous metals: fabrication strategies and advanced electrochemical applications in catalysis, sensing and energy systems. Chem. Soc. Rev. 41, 7016 (2012).

12. Kang, Y. et al. Synthesis of hierarchical porous metals using ionic-liquid-based media as solvent and template. Angew. Chem. Int. Ed. 56, 12683 (2017).

13. Kim, C., Kwon, Y. \& Lee, H. Shape effect of Ag-Ni binary nanoparticles on catalytic hydrogenation aided by surface plasmons. Chem. Commun. $\mathbf{5 1}$ 12316 (2015).

14. Adkins, H. \& Cramer, H. I. The use of nickel as a catalyst for hydrogenation. J. Am. Chem. Soc. 52, 4349 (1930).

15. Falicov, L. M. \& Somorjai, G. A. Correlation between catalytic activity and bonding and coordination number of atoms and molecules on transition metal surfaces: theory and experimental evidence. Proc. Natl Acad. Sci. USA 82 2207 (1985)

16. Corma, A. et al. Exceptional oxidation activity with size-controlled supported gold clusters of low atomicity. Nat. Chem. 5, 775 (2013).

17. Ding, Y. \& Erlebacher, J. Nanoporous metals with controlled multimodal pore size distribution. J. Am. Chem. Soc. 125, 7772 (2003).

18. Sun, L., Chien, C.-L. \& Searson, P. C. Fabrication of nanoporous nickel by electrochemical dealloying. Chem. Mater. 16, 3125 (2004).

19. Gao, Z. et al. Multiply confined nickel nanocatalysts produced by atomic layer deposition for hydrogenation reactions. Angew. Chem. Int. Ed. 54, 9006 (2015).

20. He, X., Zhong, W., Au, C.-T. \& Du, Y. Size dependence of the magnetic properties of $\mathrm{Ni}$ nanoparticles prepared by thermal decomposition method. Nanoscale Res. Lett. 8, 446 (2013).

21. Werff, J. Cvd \& Kruif, C. Gd Hard-sphere colloidal dispersions: the scaling of rheological properties with particle size, volume fraction, and shear rate. J. Rheol. 33, 421 (1989).

22. Pedireddy, S. et al. One-step synthesis of zero-dimensional hollow nanoporous gold nanoparticles with enhanced methanol electrooxidation performance. Nat. Commun. 5, 4947 (2014).

23. Bates, F. S. \& Fredrickson, G. H. Block copolymer thermodynamics: theory and experiment. Annu. Rev. Phys. Chem. 41, 525 (1990). 
24. Park, M., Harrison, C., Chaikin, P. M., Register, R. A. \& Adamson, D. H. Block copolymer lithography: periodic arrays of $\sim 10^{11}$ holes in 1 square centimeter. Science 276, 1401 (1997).

25. Thurn-Albrecht, T. et al. Nanoscopic templates from oriented block copolymer films. Adv. Mater. 12, 787 (2000).

26. Cheng, J. Y. et al. Nanoscopic templates from oriented block copolymer films. Adv. Mater. 13, 1174 (2001).

27. Zalusky, A. S., Olayo-Valles, R., Taylor, C. J. \& Hillmyer, M. A. Mesoporous polystyrene monoliths. J. Am. Chem. Soc. 123, 1519 (2001).

28. Ndoni, S., Vigild, M. E. \& Berg, R. H. Nanoporous materials with spherical and gyroid cavities created by quantitative etching of polydimethylsiloxane in polystyrene-polydimethylsiloxane block copolymers. J. Am. Chem. Soc. 125 13366 (2003).

29. Ho, R. M. et al. Solvent-induced microdomain orientation in polystyrene- $b$-poly (L-lactide) diblock copolymer thin films for nanopatterning. Polymer 46, 9362 (2005).

30. Olson, D. A., Chen, L. \& Hillmyer, M. A. Templating nanoporous polymers with ordered block copolymers. Chem. Mater. 20, 869 (2008).

31. Ho, R. M. et al. Block copolymers with a twist. J. Am. Chem. Soc. 131, 18533 (2009).

32. Hsueh, H. Y., Yao, C. T. \& Ho, R. M. Well-ordered nanohybrids and nanoporous materials from gyroid block copolymer templates. Chem. Soc. Rev. 44, 1974 (2015).

33. Crossland, E. J. W. et al. Bicontinuous double gyroid hybrid solar cell. Nano Lett. 9, 2807 (2009).

34. Vignolini, S. et al. A 3D optical metamaterial made by self-assembly. Adv. Mater. 24, OP23 (2012).
35. Kibsgaard, J., Chen, Z. B., Reinecke, B. N. \& Jaramillo, T. F. Engineering the surface structure of MoS2 to preferentially expose active edge sites for electrocatalysis. Nat. Mater. 11, 963 (2012).

36. Mallory, G. O. \& Hajdu, J. B. Electroless Plating: Fundamentals and Applications. William Andrew. (1990).

37. Hsueh, H. Y. et al. Nanoporous gyroid nickel from block copolymer templates via electroless plating. Adv. Mater. 23, 3041 (2011).

38. Jang, C. F. et al. Nanoporous gyroid platinum with high catalytic activity from block copolymer templates via electroless plating. NPG Asia Mater. 7, e170 (2015).

39. Zalusky, A. S., Olayo-Valles, R., Wolf, J. H. \& Hillmyer, M. A. Ordered nanoporous polymers from polystyrene-polylactide block copolymers. J. Am. Chem. Soc. 124, 12761 (2002).

40. Lin, T. C., Yang, K. C., Georgopanos, P., Avgeropoulos, A. \& Ho, R. M. Gyroidstructured nanoporous polymer monolith from pdms-containing block copolymers for templated synthesis. Polymer 126, 360 (2017).

41. Ito, K., Nakanishi, H. \& Ujihira, Y. Extension of the equation for the annihilation lifetime of ortho-positronium at a cavity larger than $1 \mathrm{~nm}$ in radius. J. Phys. Chem. B 103, 4555 (1999).

42. Hung, W. S. et al. Non-destructive means of probing a composite polyamide membrane for characteristic free volume, void, and chemical composition. RSC Adv. 6, 85019 (2016).

43. Hsueh, H. Y. et al. Shifting networks to achieve subgroup symmetry properties. Adv. Mater. 26, 3225 (2014).

44. Littman, H. \& Bliss, H. Hydrogenation of toluene with a raney nickel catalyst reaction kinetics. Ind. Eng. Chem. 51, 659 (1959).

45. Wang, Y., Cui, X., Deng, Y. \& Shi, F. Catalytic hydrogenation of aromatic rings catalyzed by Pd/NiO. RSC Adv. 4, 2729 (2014). 